\title{
Vanishing retinal arterial aneurysms: a case report
}

\author{
Sarah L Owens, Zdenek J Gregor
}

\begin{abstract}
An 18-year-old woman with retinal vasculitis developed multiple retinal arterial aneurysms over a period of 3 years. Subsequently she developed macular oedema, peripheral neovascularisation, and vitreous haemorrhage and was treated with systemic steroids, laser photocoagulation, and vitreoretinal surgery. No systemic cause for vasculitis was found. Serial fluorescein angiography demonstrated gradual resolution of the arterial aneurysms over the subsequent 7 years.

(Brf Ophthalmol 1992; 76: 637-638)
\end{abstract}

An 18-year-old Asian Indian woman was seen in August 1984, complaining of poor vision in the

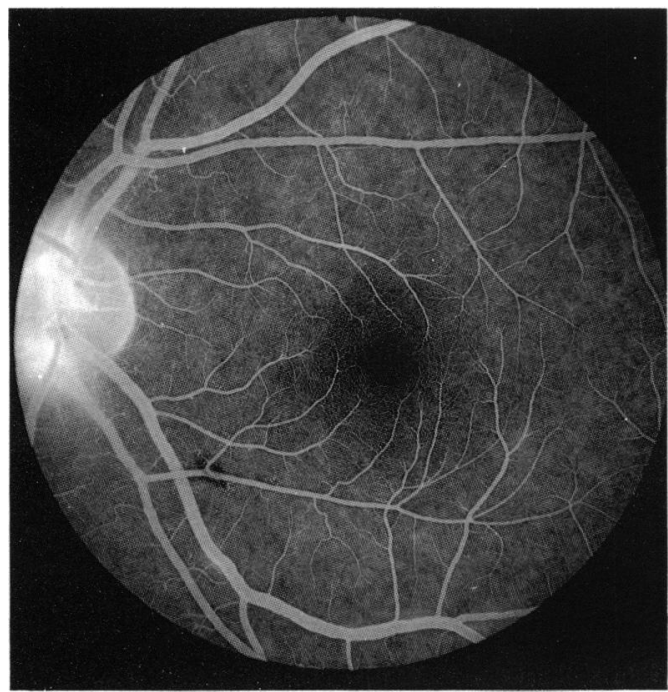

Figure 1 Fluorescein angiogram, late arteriovenous phase, left eye, 1981. Note normal calibre of retinal arterial system.

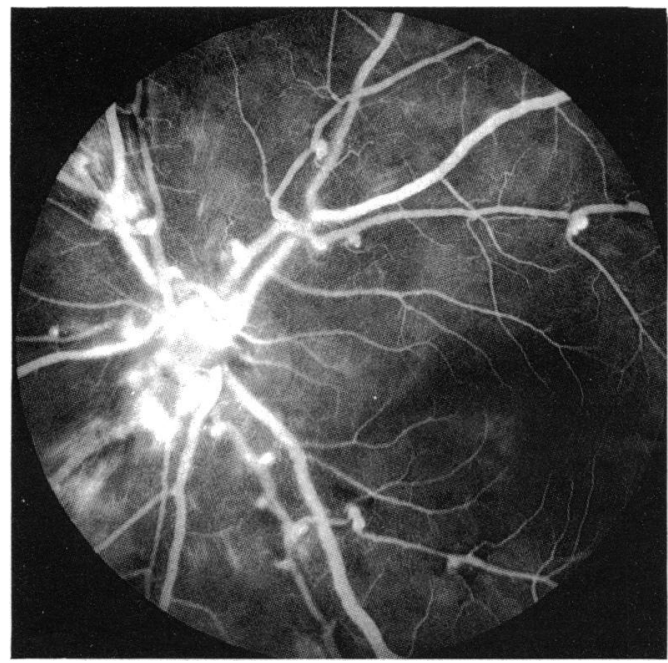

Figure 2 Fluorescein angiogram, mid arteriovenous phase, left eye, 1984. Note multiple retinal arterial aneurysms and disc neovascularisation. right eye for the past year. Her first complaint of visual difficulty occurred in 1981; at that time she was managed at another hospital for retinal vasculitis. A fluorescein angiogram from 1981 showed peripheral retinal vascular leakage but documented normal retinal vascular calibre (Fig 1).

On initial examination visual acuity was $6 / 36$ in the right eye and $6 / 6$ in the left eye. Both anterior chambers had mild cell and flare. Funduscopic examination revealed minimal disc swelling in both eyes. The most striking feature was the presence of multiple retinal arterial aneurysms (Fig 2). A fluorescein angiogram disclosed severe peripheral non perfusion (Fig 3). A comprehensive systemic work-up was unrevealing, and a diagnosis of atypical vasculitis was made.

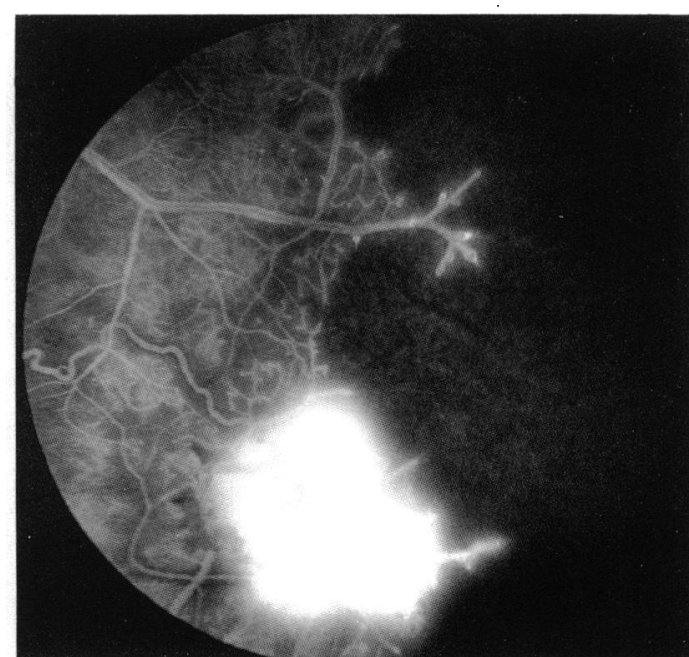

Figure 3 Fluorescein angiogram, venous phase, left eye, 1984. Note severe peripheral non perfusion with leakage from adjacent retinal neovascularisation.

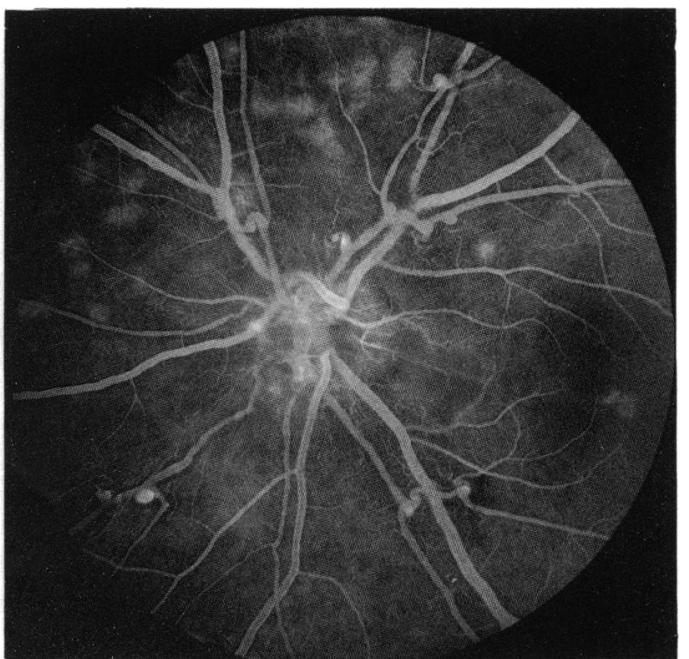

Figure 4 Fluorescein angiogram, late arteriovenous phase, left eye, 1990. Note resolution of some aneurysms, particularly of the inferotemporal branch artery when compared with Figure 2 .
Correspondence to:

Accepted for publication 23 April 1992
Moorfields Eye Hospital, City Road, London EC1V 2PD 
She was treated initially with high-dose systemic steroids and panretinal photocoagulation in both eyes. However, despite treatment her visual acuity decreased to hand movements in the right eye. She subsequently suffered a vitreous haemorrhage in the right eye; vitrectomy was performed in October 1987. By early 1988 her disease became quiescent with no medication. She reached a final visual acuity of $6 / 60$ in the right eye and $6 / 6$ in the left eye 9 years after the onset of her disease. Throughout this period there was a gradual decrease in the size and number of retinal arterial aneurysms. This was documented by the most recent angiogram (Fig 4).

\section{Discussion}

Three cases of retinal vasculitis and multiple retinal arterial aneurysms have been reported. ${ }^{12}$ The appearance and subsequent regression of these abnormalities has not been reported previously.

Aetiological considerations include infection, connective tissue disorders, degenerative retinal disease, and neoplasia. Rarer causes of vasculitis primarily affecting arteries (as in our case) include polyarteritis nodosa, systemic lupus erythematosus, Goodpasture's syndrome, Churg Strauss syndrome, and Loeffler's eosinophilic syndrome. ${ }^{3}$

As in the other cases of multiple retinal arterial aneurysms reported in the literature, our patient was young and otherwise well. A comprehensive work-up in our patient was unrevealing. No systemic disorder developed during 10 years' follow-up.

In contrast to the other reported cases our patient suffered substantial retinal exudative and ischaemic complications during the long followup period. This case also demonstrated the hitherto unreported reversibility of the aneurysms once the disease became quiescent. The underlying mechanisms for this process remain unclear.

1 Karel I, Peleska M, Disisova G. Fluorescein angiography in retinal vasculitis in children's uveitis. Ophthalmologica 1973; 166: 251-64.

2 Kincaid J, Schatz $\mathbf{H}$. Bilateral retinal areteritis with multiple aneurysmal dilatations. Retina $1983 ; 3$ : 171-8.

3 Sanders M. Duke-Elder lecture. Retinal arteritis, retinal vasculitis, and autoimmune retinal vasculitis. Eye 1987; 1: 441- 